VISION

\section{Visual Feedback}

from our Animal Behaviour Correspondent

AN ingenious series of experiments carried out on kittens during the past 10 years at the Massachusetts Institute of Technology establishing the conditions necessary for the development of normal visual-motor coordination have shown that kittens require visual feedback from the result of their own movements. Kittens moved passively through the environment without experiencing the visual consequences of moving their limbs remain ill coordinated, unable to direct their movements and apparently with little idea of the spatial configurations of the environment.

In the latest of this series of studies, A. Hein and R. M. Diamond (J. Comp. Physiol. Psychol., 81, 394 ; 1972) have attempted to separate two components of this visual feedback: "seeing arm limb movements" and "seeing environment change as result of own movement". Their results suggest that it is not sufficient for a kitten to see its limbs move in isolation from the rest of the environment ; only if the kitten has also experienced some other aspects of its visual environment in relation to its own movements will it be able to perform such tasks as reaching out a paw and touching a bar.

Hein and Diamond gave kittens experience of visual feedback from their paws in isolation from other sorts of visual experience by putting a spot of luminous paint on one paw and keeping the kittens in the dark. In this way, the only thing they could see was a patch of light as their own paw moved. Perhaps not surprisingly, after 10 days of this, the kittens gave evidence of being very ill coordinated, unable to reach out towards objects, and bumping into obstacles. They were then allowed experience of a normally illuminated room. Half of them had opaque collars on so that, although they could move freely about, they could not see their own limbs and so did not receive visual feedback from their own limb movements. The other half were not only prevented from seeing their own limbs but were restrained in a holder, so that they could only see what was going on around them. After 10 days of this treatment, none of the kittens showed visually guided reaching-that is, when they were lowered towards a ladder, they did not reach out their paws towards the rungs. But the kittens that had been allowed to move freely were superior in one respect: they avoided obstacles when moving around, unlike the restrained kittens. All kittens were then given a further 10 days of the luminous paw treatment, but this time the spot of paint was put onto the opposite paw from that in their initial exposure. As before, the only thing the kittens could see during this time was the spot of paint on their paw, as the rest of their environment was in total darkness.

It was after this treatment that the difference in reaching ability between restrained and unrestrained kittens became apparent. Only the animals that had previously developed visually guided locomotion (the ability to avoid obstacles by visual means, for example) showed properly developed visually guided reaching. What was even more remarkable was that they could reach correctly only with the paw which had had the spot of paint on it and from which they had had visual feedback, in the third part of the experiment. They were unable to guide their other paw correctly. Kittens which had been restrained and had never developed the ability to move around without bumping into things could not reach correctly with either of their paws.

Previous studies had shown that in the kitten, visual feedback from a moving limb is essential for the development of properly coordinated visually guided reaching. This experiment has added a proviso: it would seem that visual feedback from the forelimb is sufficient for the acquisition of visually guided reaching only if visually guided locomotion around the environment has also been developed.
PROTEINS

\section{Hunting the Hybrid}

from our Molecular Biology Correspondent ONE of the unswept corners of haemoglobin chemistry, which has irked the practitioners for some time, is the balance of species present in a mixture of two haemoglobins, such as occurs in sickle-cell trait blood. Electrophoretically, normal adult and sickle-cell haemoglobins, $\alpha_{2}^{\mathrm{A}} \beta_{2}^{\mathrm{A}}$ and $\alpha_{2}^{\mathrm{A}} \beta_{2}^{\mathrm{S}}$, separate easily enough, but because the haemoglobin tetramer is in rapid equilibrium with its symmetrical $\alpha \beta$ dimer, there should be every reason to expect the hybrid $\alpha_{2}^{\mathrm{A}} \beta^{\mathrm{A}} \beta^{\mathrm{S}}$ to be represented in the mixture. This, however, is an elusive creature, which escapes its pursuers by dissociating during the fractionation process: the hybrid will have an electrophoretic mobility between those of the parent species, haemoglobins $A$ and $S$. The dimers $\alpha^{\mathbf{A}} \beta^{\mathbf{A}}$ and $\alpha^{\mathbf{A}} \beta^{S}$, in equilibrium with the hybrid tetramer (no matter in how low proportion), will accordingly separate from the latter, one ahead and one behind, and turn respectively into $\alpha_{2}^{A} \beta_{2}^{A}$ and $\alpha_{2}^{A} \beta_{2}^{S}$ tetramers. Unless then the dissociation to dimers can be inhibited, the existence of the hybrid, like the extinguished light in the refrigerator when the door is shut, can only be taken on faith. Macleod and Hill (J. Biol. Chem., 248, 100; 1973) have now finally found a method, using a covalent cross-linking reagent, to salt the beast's tail.

\title{
Oncogenic Potential of Herpesvirus saimiri
}

Herpesvirus saimiri, which is indigenous to squirrel monkeys and which has not been associated with any disease, malignant or otherwise, in animals of this species, has of late attracted the attention of tumour viro. logists because when it is inoculated into other primates-marmosets, ringtail, owl and African green monkeys, for example-it induces lymphomas or leukaemias. Virtually nothing is known to date about the molecular biology of this oncogenic herpesvirus, but as Ablashi and seven colleagues report in Nature New Biology next Wednesday (March 7), the oncogenic potential of this virus remains after the virus has been heat inactivated such that it can no longer replicate and kill owl, monkey and African green monkey cells.

Ablashi et al. propagated and isolated infectious Herpesvirus saimiri in Vero cells, a stable line of cells derived from African green monkey tissue. They then inactivated the viral particles by heating them to $56^{\circ} \mathrm{C}$ for $30 \mathrm{~min}$. Intact viral particles were not morphologically changed by this treatment although the capsids of unenveloped particles appeared to collapse during heating. After this treatment the virus failed to produce any cytopathic effect in Vero cells or in owl monkey cells. Four owl monkeys, however, after receiving six weekly injections of the inactivated virus, eventually developed malignant lymphomas the pathology of which is described in some detail by Ablashi and his colleagues. Furthermore, infectious Herpesvirus saimiri could be recovered by co-cultivating, with Vero cells or owl monkey cells, tissues collected from the lymphomatous owl monkeys at necropsy.

It seems, therefore, that Herpesvirus saimiri inactivated by heat so that it is no longer able to kill susceptible host cells may retain not only the ability to transform cells and induce tumours but also retain the genetic information required to code for complete infectious progeny viral particles. The precise nature of the molecular mechanisms that underlie these phenomena remains unknown, but it seems clear that the cytocidal and oncogenic potential of this virus can be separated. 